CRYSTALLOGRAPHIC COMMUNICATIONS

ISSN 2056-9890

Received 27 December 2016

Accepted 17 January 2017

Edited by M. Weil, Vienna University of Technology, Austria

Keywords: crystal structure; molecular salt; oxonium ion; hexacyanidoferrate(II); ethylpyridinium ion; hydrogen bonds.

CCDC reference: 1527928

Supporting information: this article has supporting information at journals.iucr.org/e

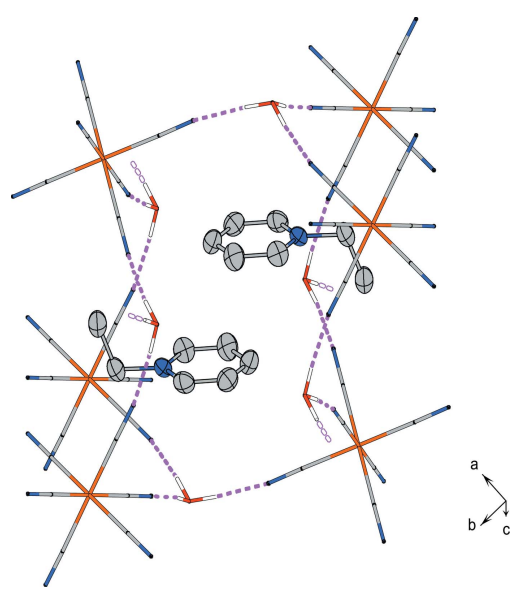

OPEN $\odot$ ACCESS

\section{Crystal structure of bis(1-ethylpyridinium) dioxonium hexacyanidoferrate(II)}

\author{
Rikako Tanaka and Nobuyuki Matsushita*
}

Department of Chemistry and Research Center for Smart Molecules, Rikkyo University, Nishi-Ikebukuro 3-34-1, Toshimaku, 171-8501 Tokyo, Japan. *Correspondence e-mail: cnmatsu@rikkyo.ac.jp

The title compound, $\left(\mathrm{C}_{7} \mathrm{H}_{10} \mathrm{~N}\right)_{2}\left(\mathrm{H}_{3} \mathrm{O}\right)_{2}\left[\mathrm{Fe}(\mathrm{CN})_{6}\right]$ or $(\text { Etpy })_{2}\left(\mathrm{H}_{3} \mathrm{O}\right)_{2}\left[\mathrm{Fe}(\mathrm{CN})_{6}\right]$ (Etpy ${ }^{+}$is 1-ethylpyridinium), crystallizes in the space group Pnnm. The $\mathrm{Fe}^{\mathrm{II}}$ atom of the $\left[\mathrm{Fe}(\mathrm{CN})_{6}\right]^{4-}$ anion lies on a site with site symmetry ..2/m, and has an octahedral coordination sphere defined by six cyanido ligands. Both the Etpy ${ }^{+}$ and the oxonium cations are located on a mirror plane. In the crystal, electrondonor anions of $\left[\mathrm{Fe}(\mathrm{CN})_{6}\right]^{4-}$ and electron-acceptor cations of Etpy ${ }^{+}$are each stacked parallel to the $b$ axis, resulting in a columnar structure with segregated moieties. The crystal packing is stabilized by a three-dimensional $\mathrm{O}-\mathrm{H} \cdots \mathrm{N}$ hydrogen-bonding network between the oxonium ions and the cyanide ligands of $\left[\mathrm{Fe}(\mathrm{CN})_{6}\right]^{4-}$.

\section{Chemical context}

Prussian blue is a well-known compound which displays a deep-blue colour based on an intervalence charge-transfer interaction between $\left[\mathrm{Fe}^{\mathrm{II}}(\mathrm{CN})_{6}\right]^{4-}$ electron-donor species and $\mathrm{Fe}^{\mathrm{III}}$ electron-acceptor species. Several charge-transfer salts composed of $\left[\mathrm{Fe}(\mathrm{CN})_{6}\right]^{4-}$ and organic acceptor cations, e.g. 1,1'-dimethyl-4,4'-bipyridinium (methyl viologen) have been reported (Nakahara \& Wang, 1963; Kostina et al., 2001; Kotov et al., 2005; Abouelwafa et al., 2010). In the majority of cases, the reported charge-transfer salts of $\left[\mathrm{Fe}(\mathrm{CN})_{6}\right]^{4-}$ are accompanied by dicationic organic acceptor species. On the other hand, charge-transfer salts of $\left[\mathrm{Fe}(\mathrm{CN})_{6}\right]^{4-}$ accompanied by monocationic species are rather rare (Gorelsky et al., 2007).<smiles>[Z]C1CCCCC1</smiles><smiles></smiles>

The present X-ray crystallographic analysis of the title salt, (Etpy) $)_{2}\left(\mathrm{H}_{3} \mathrm{O}\right)_{2}\left[\mathrm{Fe}(\mathrm{CN})_{6}\right]\left(\right.$ Etpy $^{+}$is 1-ethylpyridinium), (I), was performed in order to elucidate the crystal packing of a charge-transfer hexacyanidoferrate(II) anion with a monocationic organic acceptor and an oxonium ion.

\section{Structural commentary}

The structures of the molecular components of (I) are displayed in Fig. 1. The asymmetric unit of (I) contains half of an Etpy ${ }^{+}$cation and an oxonium ion (both located on a mirror plane), and one quarter of an $\left[\mathrm{Fe}(\mathrm{CN})_{6}\right]^{4-}$ anion, the $\mathrm{Fe}^{\mathrm{II}}$ atom 


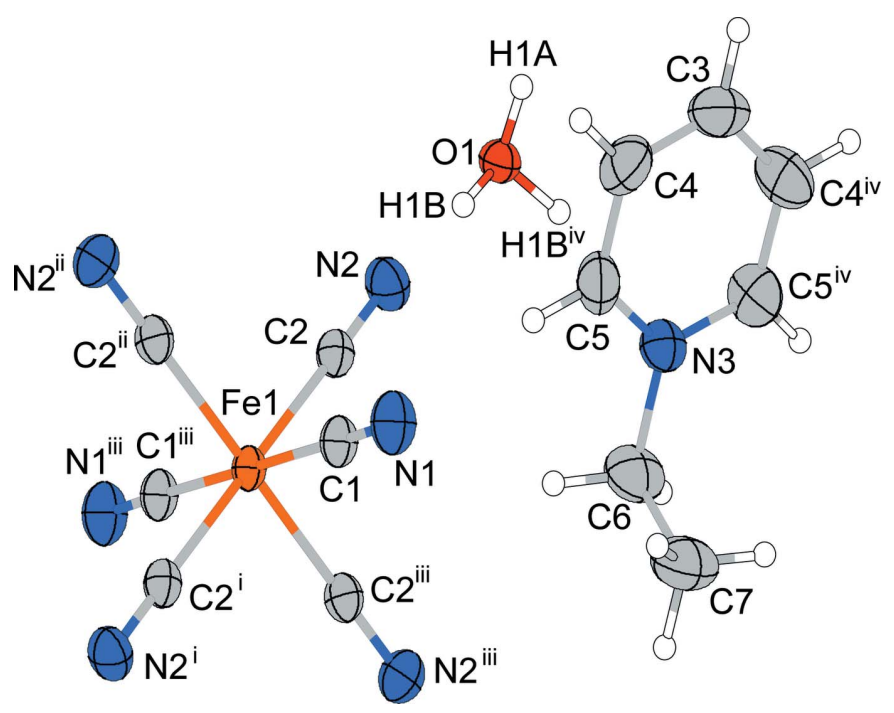

Figure 1

The structures of the molecular components of compound (I), showing the atomic numbering scheme. Displacement ellipsoids are drawn at the $50 \%$ probability level for non-H atoms. [Symmetry codes: (i) $-x,-y,-z$; (ii) $x, y,-z$; (iii) $-x,-y, z$; (iv) $x, y,-z+1$.]

of which is located on a site with symmetry $. .2 / m$. The $\mathrm{Fe}^{\mathrm{II}}$ atom is coordinated by six cyanido ligands in a slightly distorted octahedral configuration $[\mathrm{Fe}-\mathrm{C}=1.9045(18)$, 1.9068 (13) $\AA$; $\mathrm{C} \equiv \mathrm{N}=1.157$ (2), 1.1598 (17) $\AA$; $\mathrm{C}-\mathrm{Fe}-\mathrm{C}_{\text {trans }}$ $=180.0^{\circ} ; \mathrm{C}-\mathrm{Fe}-\mathrm{C}_{c i s}=89.60(7)-90.40(7)^{\circ} ; \mathrm{Fe}-\mathrm{C}-\mathrm{N}=$ $\left.178.67(18), 179.77(13)^{\circ}\right]$. The bond angle of the ethyl group of the $\mathrm{Etpy}^{+}$ion $\left[\mathrm{N} 3-\mathrm{C} 6-\mathrm{C} 7=110.77(19)^{\circ}\right]$ is similar to those of Etpy[ $\left.\mathrm{AlCl}_{4}\right]$ [109.2 (11) $)^{\circ}$ Z Zaworotko et al., 1989] and poly[4-dimethylamino-1-ethylpyridin-1-ium [tri- $\mu$-dicyanamido- $\kappa^{6} N^{1}: N^{5}$-cadmium]] [111.5 (5) ${ }^{\circ}$; Wang et al., 2015].

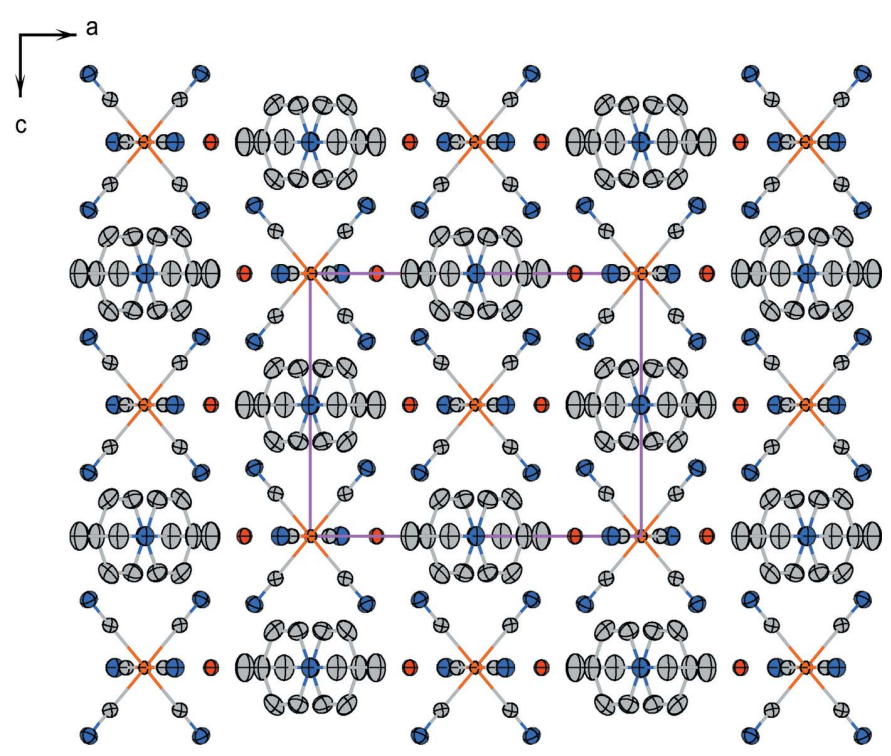

Figure 2

The crystal packing of compound (I) in a view along the $b$ axis. $\mathrm{H}$ atoms have been omitted for clarify; the probability function is as in Fig. 1.
Table 1

Hydrogen-bond geometry $\left(\AA,^{\circ}\right)$.

\begin{tabular}{lllll}
\hline$D-\mathrm{H} \cdots A$ & $D-\mathrm{H}$ & $\mathrm{H} \cdots A$ & $D \cdots A$ & $D-\mathrm{H} \cdots A$ \\
\hline $\mathrm{O} 1-\mathrm{H} 1 A \cdots \mathrm{N} 1^{\mathrm{i}}$ & $0.90(3)$ & $1.67(3)$ & $2.569(2)$ & $176(3)$ \\
$\mathrm{O} 1-\mathrm{H} 1 B \cdots \mathrm{N} 2$ & $0.931(18)$ & $1.632(19)$ & $2.5589(15)$ & $173.6(18)$ \\
\hline
\end{tabular}

Symmetry code: (i) $x+\frac{1}{2},-y+\frac{1}{2},-z+\frac{1}{2}$.

\section{Supramolecular features}

The projection of the crystal structure of (I) along the $b$ axis is shown in Fig. 2.

The $\left[\mathrm{Fe}(\mathrm{CN})_{6}\right]^{4-}$ electron-donor anions and the Etpy ${ }^{+}$ electron-acceptor cations stack separately in columns parallel to the $b$ axis whereby both types of columns are alternately arranged in the $a$ - and $c$-axis directions.

In the crystal of (I), the oxonium ions and $\left[\mathrm{Fe}(\mathrm{CN})_{6}\right]^{4-}$ ions form a three-dimensional $\mathrm{O}-\mathrm{H} \cdots \mathrm{N}$ hydrogen-bonding network (Table 1). A pair of Etpy ${ }^{+}$cations is enclosed in the hydrogen-bonding cage formed by six $\left[\mathrm{Fe}(\mathrm{CN})_{6}\right]^{4-}$ ions and six oxonium ions (Fig. 3). Two pyridinium rings of the Etpy ${ }^{+}$ cations are arranged in parallel and the ethyl groups are alternating with each other. The centroid-to-centroid distance (4.147 $\AA$ ) and the face-to-face distance of the least-square planes $(3.731 \AA$ ) between two pyridinium rings indicate that $\pi-\pi$ interactions are not developed.

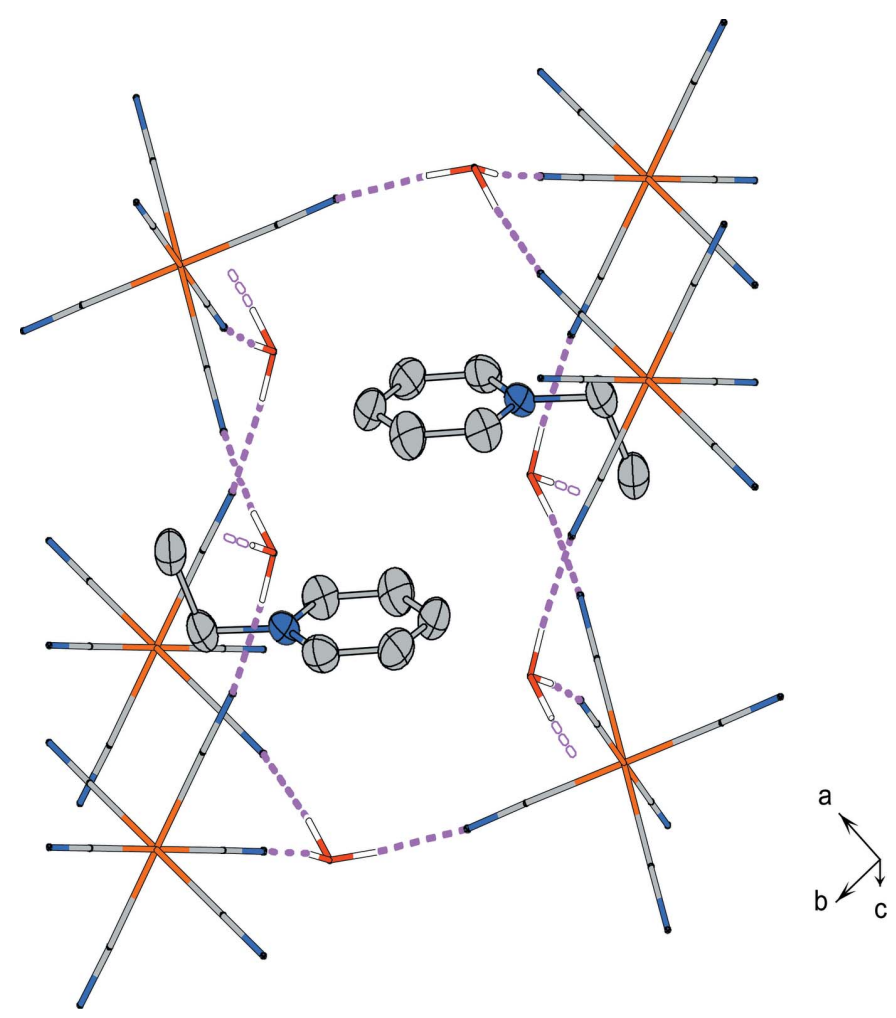

Figure 3

Hydrogen-bonding network composed of $\left[\mathrm{Fe}(\mathrm{CN})_{6}\right]^{4-}$ anions and oxonium cations. Magenta dashed lines represent hydrogen bonds. 


\section{Database survey}

Several crystal structures of compounds containing the Etpy ${ }^{+}$ cation have been deposited in the Cambridge Structural Database (Groom et al. 2016), e.g. Etpy[ $\left.\mathrm{AlCl}_{4}\right]$ (Zaworotko et al., 1989), Etpy[Ni(mnt) $\left.)_{2}\right](\mathrm{mnt}=$ maleonitrile-1,2-dithiolate; Robertson et al., 1999), or (Etpy $)_{2}\left[\mathrm{CoCl}_{4}\right]$ (Felloni et al., 2004). A hexacyanidoferrate(II) salt, $(\mathrm{Hpy})_{2}\left(\mathrm{H}_{3} \mathrm{O}\right)_{2}\left[\mathrm{Fe}(\mathrm{CN})_{6}\right]\left(\mathrm{Hpy}^{+}\right.$ $=N$-hydropyridinium; Gorelsky et al., 2007), quite similar to (I), has been also reported.

\section{Synthesis and crystallization}

$\mathrm{H}_{4}\left[\mathrm{Fe}(\mathrm{CN})_{6}\right]$ (106 mg) and L-ascorbic acid (60 mg) were dissolved in water $(17 \mathrm{ml})$. The mixture was added to an aqueous solution of 1-ethylpyridinium bromide (177 mg/ $17 \mathrm{ml}$ ). After standing at $277 \mathrm{~K}$ for a day, yellow plateletshaped crystals suitable for X-ray analysis were obtained. Elemental analysis: found: C, 51.52; H, 5.878; N, 24.06\%; calculated for $\mathrm{C}_{20} \mathrm{H}_{26} \mathrm{FeN}_{8} \mathrm{O}_{2}$ : C, 51.51; H, 5.63; N, $24.03 \%$. Thermogravimetry was measured from 296 to $476 \mathrm{~K}$ at a rate of $5 \mathrm{~K} \mathrm{~min}^{-1}$ under $\mathrm{N}_{2}$ gas flow $\left(100 \mathrm{ml} \mathrm{min}^{-1}\right)$ on a Rigaku TG-DTA Thermo Plus EVO2 TG8121. Found: $7.85 \%$ mass loss; calculated: $7.73 \%$. The mass loss of (I) took place at around 373 to $393 \mathrm{~K}$ and corresponds to two water molecules per chemical formula. The result suggests that the water molecules are released from the oxonium ions. Most probably, protons, $\mathrm{H}^{+}$, remain in the crystal as counter-cations. The IR spectrum of compound (I) is shown in Fig. 4. Selected IR bands ( $\mathrm{KBr}$ pellet, $\left.\mathrm{cm}^{-1}\right)$ : 3135-2941 ( $\left.s, \mathrm{C}-\mathrm{H}, \mathrm{str}\right), 2640(\mathrm{br}$, $\mathrm{O}-\mathrm{H}, s t r), 2075(s, \mathrm{C} \equiv \mathrm{N}, s t r)$.

\section{Refinement}

Crystal data, data collection and structure refinement details are summarized in Table 2. In the final refinement of the title compound, three reflections, viz. (0 17 1), (2 16 0) and (5 15 2),

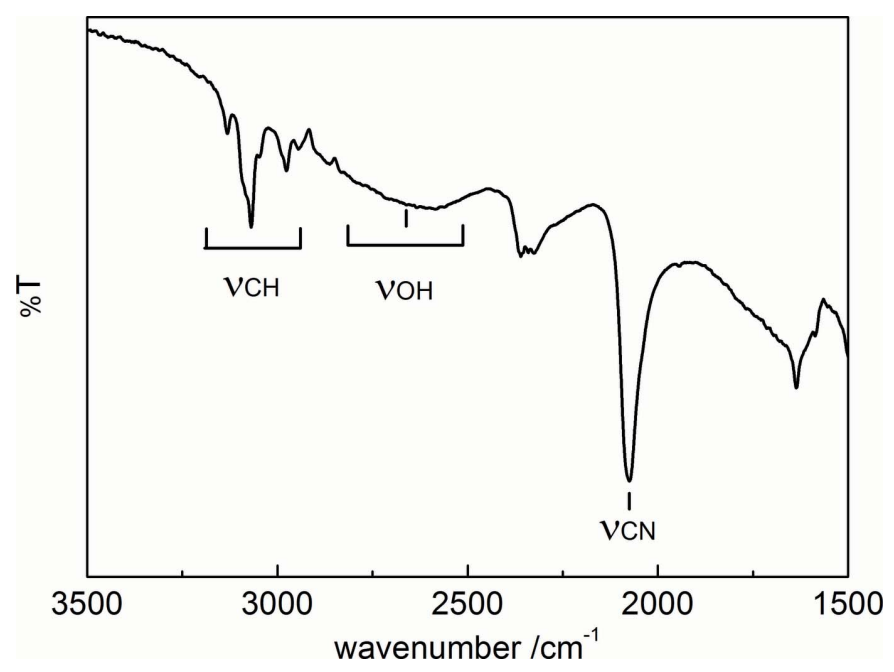

Figure 4

The IR spectrum of compound (I).
Table 2

Experimental details.

\begin{tabular}{ll}
\hline Crystal data & \\
Chemical formula & $\left(\mathrm{C}_{7} \mathrm{H}_{10} \mathrm{~N}\right)_{2}\left(\mathrm{H}_{3} \mathrm{O}\right)_{2}\left[\mathrm{Fe}(\mathrm{CN})_{6}\right]$ \\
$M_{\mathrm{r}}$ & 466.34 \\
Crystal system, space group & Orthorhombic, Pnnm \\
Temperature $(\mathrm{K})$ & 173 \\
$a, b, c(\AA)$ & $11.8807(4), 12.1279(7), 8.3962(2)$ \\
$V\left(\AA^{3}\right)$ & $1209.79(9)$ \\
$Z$ & 2 \\
Radiation type & Mo $K \alpha$ \\
$\mu\left(\mathrm{mm}^{-1}\right)$ & 0.65 \\
Crystal size $(\mathrm{mm})$ & $0.28 \times 0.13 \times 0.08$ \\
& \\
Data collection & Rigaku R-AXIS RAPID imaging- \\
Diffractometer & plate \\
& Multi-scan $(A B S C O R ;$ Higashi, \\
Absorption correction & $1995)$ \\
& $0.907,0.952$ \\
$T_{\min }, T_{\max }$ & $25575,2216,1840$ \\
No. of measured, independent and & \\
$\quad$ observed $[I>2 \sigma(I)]$ reflections & 0.034 \\
$R_{\text {int }}$ & 0.746 \\
(sin $\theta / \lambda)_{\text {max }}\left(\AA^{-1}\right)$ & \\
& \\
Refinement & \\
$R\left[F^{2}>2 \sigma\left(F^{2}\right)\right], w R\left(F^{2}\right), S$ & $0.036,0.093,1.11$ \\
No. of reflections & 2213 \\
No. of parameters & 88 \\
H-atom treatment & H atoms treated by a mixture of \\
& independent and constrained \\
$\Delta \rho_{\text {max }}, \Delta \rho_{\text {min }}\left(\mathrm{e} \AA^{-3}\right)$ & refinement \\
\hline & $0.50,-0.70$ \\
\hline
\end{tabular}

Computer programs: RAPID-AUTO (Rigaku, 2006), SHELXT2014 (Sheldrick, 2015a), SHELXL2014 (Sheldrick, 2015b), DIAMOND (Brandenburg, 2016) and publCIF (Westrip, 2010).

were omitted owing to poor agreements between observed and calculated intensities. $\mathrm{H}$ atoms of the Etpy ${ }^{+}$cation were, at first, located in a difference Fourier map, but finally placed in geometrically calculated positions and refined as riding, with $\mathrm{C}$ (methylene $)-\mathrm{H}=0.92 \AA, \mathrm{C}($ methyl $)-\mathrm{H}=0.98 \AA$ and $\mathrm{C}($ aromatic $)-\mathrm{H}=0.95 \AA$, all with $U_{\text {iso }}(\mathrm{H})=1.5 U_{\text {eq }}(\mathrm{C}) . \mathrm{H}$ atoms of the oxonium ion were located in a difference Fourier map and their positions refined with $U_{\text {iso }}(\mathrm{H})=1.5 U_{\text {eq }}(\mathrm{O})$. The maximum and minimum electron density peaks are located $1.00 \AA$ from atom $\mathrm{C} 1$ and $0.71 \AA$ from atom Fe 1 , respectively.

\section{Acknowledgements}

The authors would acknowledge a Special Fund for Research (SFR) from Rikkyo University.

\section{References}

Abouelwafa, A. S., Mereacre, V., Balaban, T. S., Anson, C. E. \& Powell, A. K. (2010). CrystEngComm, 12, 94-99.

Brandenburg, K. (2016). DIAMOND. Crystal Impact GbR, Bonn, Germany.

Felloni, M., Hubberstey, P., Wilson, C. \& Schröder, M. (2004). CrystEngComm, 6, 87-95.

Gorelsky, S. I., Ilyukhin, A. B., Kholin, P. V., Kotov, V. Y., Lokshin, B. V. \& Sapoletova, N. V. (2007). Inorg. Chim. Acta, 360, 2573-2582.

Groom, C. R., Bruno, I. J., Lightfoot, M. P. \& Ward, S. C. (2016). Acta Cryst. B72, 171-179.

Higashi, T. (1995). ABSCOR. Rigaku Corporation, Tokyo, Japan. 
Kostina, S. A., Ilyukhin, A. B., Lokshin, B. V. \& Kotov, V. Y. (2001). Mendeleev Commun. 11, 12-13.

Kotov, V. Y., Ilyukhin, A. B., Lunina, V. K. \& Shpigun, L. K. (2005). Mendeleev Commun. 15, 95-96.

Nakahara, A. \& Wang, J. H. (1963). J. Phys. Chem. 67, 496-498.

Rigaku (2006). RAPID-AUTO. Rigaku Corporation, Tokyo, Japan.

Robertson, N., Bergemann, C., Becker, H., Agarwal, P., Julian, S. R., Friend, R. H., Hatton, N. J., Underhill, A. E. \& Kobayashi, A. (1999). J. Mater. Chem. 9, 1713-1717.
Sheldrick, G. M. (2015a). Acta Cryst. A71, 3-8.

Sheldrick, G. M. (2015b). Acta Cryst. C71, 3-8.

Wang, H.-T., Zhou, L. \& Wang, X.-L. (2015). Acta Cryst. C71, 717720.

Westrip, S. P. (2010). J. Appl. Cryst. 43, 920-925.

Zaworotko, M. J., Cameron, T. S., Linden, A. \& Sturge, K. C. (1989). Acta Cryst. C45, 996-1002. 


\section{supporting information}

Acta Cryst. (2017). E73, 219-222 [https://doi.org/10.1107/S2056989017000810]

\section{Crystal structure of bis(1-ethylpyridinium) dioxonium hexacyanidoferrate(II)}

\section{Rikako Tanaka and Nobuyuki Matsushita}

\section{Computing details}

Data collection: RAPID-AUTO (Rigaku, 2006); cell refinement: RAPID-AUTO (Rigaku, 2006); data reduction: RAPIDAUTO (Rigaku, 2006); program(s) used to solve structure: SHELXT2014 (Sheldrick, 2015a); program(s) used to refine structure: SHELXL2014 (Sheldrick, 2015b); molecular graphics: DIAMOND (Brandenburg, 2016); software used to prepare material for publication: publCIF (Westrip, 2010).

Bis(1-ethylpyridinium) dioxonium hexacyanidoferrate(II)

Crystal data

$\left(\mathrm{C}_{7} \mathrm{H}_{10} \mathrm{~N}\right)_{2}\left(\mathrm{H}_{3} \mathrm{O}\right)_{2}\left[\mathrm{Fe}(\mathrm{CN})_{6}\right]$

$M_{r}=466.34$

Orthorhombic, Pnnm

$a=11.8807$ (4) $\AA$

$b=12.1279(7) \AA$

$c=8.3962(2) \AA$

$V=1209.79(9) \AA^{3}$

$Z=2$

$F(000)=488$

\section{Data collection}

Rigaku R-AXIS RAPID imaging-plate diffractometer

Radiation source: X-ray sealed tube

Graphite monochromator

Detector resolution: 10.00 pixels $\mathrm{mm}^{-1}$

$\omega$ scans

Absorption correction: multi-scan (ABSCOR; Higashi, 1995)

$T_{\min }=0.907, T_{\max }=0.952$

\section{Refinement}

Refinement on $F^{2}$

Least-squares matrix: full

$R\left[F^{2}>2 \sigma\left(F^{2}\right)\right]=0.036$

$w R\left(F^{2}\right)=0.093$

$S=1.11$

2213 reflections

88 parameters

0 restraints

Primary atom site location: structure-invariant direct methods
$D_{\mathrm{x}}=1.280 \mathrm{Mg} \mathrm{m}^{-3}$

Mo $K \alpha$ radiation, $\lambda=0.7107 \AA$

Cell parameters from 8639 reflections

$\theta=3.4-32.0^{\circ}$

$\mu=0.65 \mathrm{~mm}^{-1}$

$T=173 \mathrm{~K}$

Block, pale-yellow

$0.28 \times 0.13 \times 0.08 \mathrm{~mm}$

25575 measured reflections

2216 independent reflections

1840 reflections with $I>2 \sigma(I)$

$R_{\text {int }}=0.034$

$\theta_{\max }=32.0^{\circ}, \theta_{\min }=3.4^{\circ}$

$h=-17 \rightarrow 17$

$k=-18 \rightarrow 18$

$l=-12 \rightarrow 10$

Secondary atom site location: difference Fourier map

Hydrogen site location: difference Fourier map

$\mathrm{H}$ atoms treated by a mixture of independent and constrained refinement

$w=1 /\left[\sigma^{2}\left(F_{\mathrm{o}}^{2}\right)+(0.0356 P)^{2}+0.6061 P\right]$

where $P=\left(F_{\mathrm{o}}^{2}+2 F_{\mathrm{c}}^{2}\right) / 3$

$(\Delta / \sigma)_{\max }<0.001$

$\Delta \rho_{\max }=0.50 \mathrm{e} \AA^{-3}$

$\Delta \rho_{\min }=-0.70$ e $\AA^{-3}$ 


\section{Special details}

Geometry. All esds (except the esd in the dihedral angle between two 1.s. planes) are estimated using the full covariance matrix. The cell esds are taken into account individually in the estimation of esds in distances, angles and torsion angles; correlations between esds in cell parameters are only used when they are defined by crystal symmetry. An approximate (isotropic) treatment of cell esds is used for estimating esds involving l.s. planes.

Fractional atomic coordinates and isotropic or equivalent isotropic displacement parameters $\left(\hat{A}^{2}\right)$

\begin{tabular}{lllll}
\hline & $x$ & $y$ & $z$ & $U_{\text {iso }} / U_{\text {eq }}$ \\
\hline Fe1 & 0.0000 & 0.0000 & 0.0000 & $0.02095(10)$ \\
C1 & $-0.05743(16)$ & $0.14661(15)$ & 0.0000 & $0.0257(3)$ \\
C2 & $0.10536(11)$ & $0.04026(10)$ & $0.16115(15)$ & $0.0255(2)$ \\
N1 & $-0.09019(17)$ & $0.23642(15)$ & 0.0000 & $0.0371(4)$ \\
N2 & $0.16963(11)$ & $0.06441(11)$ & $0.25910(15)$ & $0.0352(3)$ \\
N3 & $0.00181(15)$ & $0.25836(14)$ & 0.5000 & $0.0333(3)$ \\
C3 & $0.1469(2)$ & $0.4327(2)$ & 0.5000 & $0.0485(6)$ \\
H3 & 0.1978 & 0.4930 & 0.5000 & $0.073^{*}$ \\
C4 & $0.10987(15)$ & $0.38888(16)$ & $0.3591(2)$ & $0.0498(4)$ \\
H4 & 0.1344 & 0.4192 & 0.2607 & $0.075^{*}$ \\
C5 & $0.03717(15)$ & $0.30083(14)$ & $0.3608(2)$ & $0.0431(4)$ \\
H5 & 0.0118 & 0.2698 & 0.2633 & $0.065^{*}$ \\
C6 & $-0.0794(2)$ & $0.16567(19)$ & 0.5000 & $0.0485(6)$ \\
H6A & -0.0670 & 0.1193 & 0.5954 & $0.073^{*}$ \\
C7 & $-0.1981(2)$ & $0.2077(2)$ & 0.5000 & $0.0570(7)$ \\
H7A & -0.2503 & 0.1452 & 0.5000 & $0.086^{*}$ \\
H7B & -0.2108 & 0.2527 & 0.5953 & $0.086^{*}$ \\
O1 & $0.30049(12)$ & $0.08078(12)$ & 0.5000 & $0.0279(3)$ \\
H1A & $0.342(2)$ & $0.143(2)$ & 0.5000 & $0.042^{*}$ \\
H1B & $0.2513(15)$ & $0.0800(15)$ & $0.414(2)$ & $0.042^{*}$
\end{tabular}

Atomic displacement parameters $\left(\AA^{2}\right)$

\begin{tabular}{lllllll}
\hline & $U^{11}$ & $U^{22}$ & $U^{33}$ & $U^{12}$ & $U^{13}$ & $U^{23}$ \\
\hline Fe1 & $0.02418(17)$ & $0.02126(16)$ & $0.01740(15)$ & $0.00227(12)$ & 0.000 & 0.000 \\
C1 & $0.0302(8)$ & $0.0250(8)$ & $0.0218(7)$ & $0.0048(6)$ & 0.000 & 0.000 \\
C2 & $0.0299(6)$ & $0.0246(5)$ & $0.0220(5)$ & $0.0015(4)$ & $0.0015(5)$ & $-0.0001(4)$ \\
N1 & $0.0454(10)$ & $0.0307(8)$ & $0.0353(9)$ & $0.0104(7)$ & 0.000 & 0.000 \\
N2 & $0.0383(6)$ & $0.0393(6)$ & $0.0280(5)$ & $-0.0029(5)$ & $-0.0044(5)$ & $-0.0030(5)$ \\
N3 & $0.0364(8)$ & $0.0254(7)$ & $0.0380(9)$ & $0.0019(6)$ & 0.000 & 0.000 \\
C3 & $0.0341(11)$ & $0.0473(13)$ & $0.0641(16)$ & $-0.0065(10)$ & 0.000 & 0.000 \\
C4 & $0.0518(9)$ & $0.0524(9)$ & $0.0451(9)$ & $-0.0064(8)$ & $0.0172(8)$ & $0.0013(8)$ \\
C5 & $0.0522(9)$ & $0.0434(8)$ & $0.0336(7)$ & $-0.0007(7)$ & $0.0058(7)$ & $-0.0083(7)$ \\
C6 & $0.0506(13)$ & $0.0276(10)$ & $0.0674(17)$ & $-0.0047(9)$ & 0.000 & 0.000 \\
C7 & $0.0469(14)$ & $0.0416(13)$ & $0.083(2)$ & $-0.0110(11)$ & 0.000 & 0.000 \\
O1 & $0.0292(6)$ & $0.0287(6)$ & $0.0257(6)$ & $-0.0063(5)$ & 0.000 & 0.000 \\
\hline
\end{tabular}


Geometric parameters $\left(\AA,{ }^{\circ}\right)$

\begin{tabular}{|c|c|c|c|}
\hline $\mathrm{Fe} 1-\mathrm{C}^{\mathrm{i}}$ & $1.9045(18)$ & $\mathrm{C} 3-\mathrm{C} 4^{\mathrm{iv}}$ & $1.369(2)$ \\
\hline $\mathrm{Fe} 1-\mathrm{C} 1$ & $1.9045(18)$ & $\mathrm{C} 3-\mathrm{H} 3$ & 0.9500 \\
\hline $\mathrm{Fe} 1-\mathrm{C} 2^{\mathrm{i}}$ & $1.9068(13)$ & $\mathrm{C} 4-\mathrm{C} 5$ & $1.373(2)$ \\
\hline $\mathrm{Fe} 1-\mathrm{C}^{2 \mathrm{ii}}$ & $1.9068(13)$ & $\mathrm{C} 4-\mathrm{H} 4$ & 0.9500 \\
\hline $\mathrm{Fe} 1-\mathrm{C} 2^{\mathrm{iii}}$ & $1.9068(13)$ & $\mathrm{C} 5-\mathrm{H} 5$ & 0.9500 \\
\hline $\mathrm{Fe} 1-\mathrm{C} 2$ & $1.9069(13)$ & $\mathrm{C} 6-\mathrm{C} 7$ & $1.499(4)$ \\
\hline $\mathrm{C} 1-\mathrm{N} 1$ & $1.157(2)$ & C6-H6A & 0.9900 \\
\hline $\mathrm{C} 2-\mathrm{N} 2$ & $1.1598(17)$ & $\mathrm{C} 7-\mathrm{H} 7 \mathrm{~A}$ & 0.9800 \\
\hline $\mathrm{N} 3-\mathrm{C} 5^{\mathrm{iv}}$ & $1.3447(19)$ & $\mathrm{C} 7-\mathrm{H} 7 \mathrm{~B}$ & 0.9800 \\
\hline $\mathrm{N} 3-\mathrm{C} 5$ & $1.3447(19)$ & $\mathrm{O} 1-\mathrm{H} 1 \mathrm{~A}$ & $0.90(3)$ \\
\hline $\mathrm{N} 3-\mathrm{C} 6$ & $1.482(3)$ & $\mathrm{O} 1-\mathrm{H} 1 \mathrm{~B}$ & $0.931(18)$ \\
\hline $\mathrm{C} 3-\mathrm{C} 4$ & $1.369(2)$ & $\mathrm{O} 1-\mathrm{H}_{1} \mathrm{~B}^{\mathrm{iv}}$ & $0.931(18)$ \\
\hline $\mathrm{C} 1-\mathrm{Fe} 1-\mathrm{C} 1$ & 180.0 & $\mathrm{C} 5-\mathrm{N} 3-\mathrm{C} 6$ & $119.61(10)$ \\
\hline $\mathrm{C} 1^{\mathrm{i}}-\mathrm{Fe} 1-\mathrm{C} 2^{\mathrm{i}}$ & $89.78(5)$ & $\mathrm{C} 4-\mathrm{C} 3-\mathrm{C} 4^{\mathrm{iv}}$ & $119.5(2)$ \\
\hline $\mathrm{C} 1-\mathrm{Fe} 1-\mathrm{C} 2^{\mathrm{i}}$ & $90.22(5)$ & $\mathrm{C} 4-\mathrm{C} 3-\mathrm{H} 3$ & 120.2 \\
\hline $\mathrm{C} 1^{\mathrm{i}}-\mathrm{Fe} 1-\mathrm{C} 2^{\mathrm{ii}}$ & $90.22(5)$ & $\mathrm{C} 4^{\mathrm{iv}}-\mathrm{C} 3-\mathrm{H} 3$ & 120.2 \\
\hline $\mathrm{C} 1-\mathrm{Fe} 1-\mathrm{C} 2^{\mathrm{ii}}$ & $89.78(5)$ & $\mathrm{C} 3-\mathrm{C} 4-\mathrm{C} 5$ & $119.65(17)$ \\
\hline $\mathrm{C} 2^{\mathrm{i}}-\mathrm{Fe} 1-\mathrm{C} 2^{\mathrm{ii}}$ & $89.60(7)$ & $\mathrm{C} 3-\mathrm{C} 4-\mathrm{H} 4$ & 120.2 \\
\hline $\mathrm{C} 1^{\mathrm{i}}-\mathrm{Fe} 1-\mathrm{C} 2^{\mathrm{iii}}$ & $89.78(5)$ & $\mathrm{C} 5-\mathrm{C} 4-\mathrm{H} 4$ & 120.2 \\
\hline $\mathrm{C} 1-\mathrm{Fe} 1-\mathrm{C} 2^{\mathrm{iii}}$ & $90.22(5)$ & $\mathrm{N} 3-\mathrm{C} 5-\mathrm{C} 4$ & $120.20(16)$ \\
\hline $\mathrm{C} 2^{\mathrm{i}}-\mathrm{Fe} 1-\mathrm{C} 2^{\mathrm{iii}}$ & $90.40(7)$ & $\mathrm{N} 3-\mathrm{C} 5-\mathrm{H} 5$ & 119.9 \\
\hline $\mathrm{C} 2^{\mathrm{ii}}-\mathrm{Fe} 1-\mathrm{C}^{\mathrm{iii}}$ & $180.00(11)$ & $\mathrm{C} 4-\mathrm{C} 5-\mathrm{H} 5$ & 119.9 \\
\hline $\mathrm{C} 1-\mathrm{Fe} 1-\mathrm{C} 2$ & $90.22(5)$ & $\mathrm{N} 3-\mathrm{C} 6-\mathrm{C} 7$ & 110.77 (19) \\
\hline $\mathrm{C} 1-\mathrm{Fe} 1-\mathrm{C} 2$ & $89.78(5)$ & N3-C6-H6A & 109.5 \\
\hline $\mathrm{C} 2 \mathrm{i}-\mathrm{Fe} 1-\mathrm{C} 2$ & 180.0 & $\mathrm{C} 7-\mathrm{C} 6-\mathrm{H} 6 \mathrm{~A}$ & 109.5 \\
\hline $\mathrm{C} 2{ }^{\mathrm{ii}}-\mathrm{Fe} 1-\mathrm{C} 2$ & $90.40(7)$ & $\mathrm{C} 6-\mathrm{C} 7-\mathrm{H} 7 \mathrm{~A}$ & 109.4 \\
\hline $\mathrm{C} 2{ }^{\mathrm{iii}}-\mathrm{Fe} 1-\mathrm{C} 2$ & $89.60(7)$ & $\mathrm{C} 6-\mathrm{C} 7-\mathrm{H} 7 \mathrm{~B}$ & 109.5 \\
\hline $\mathrm{N} 1-\mathrm{C} 1-\mathrm{Fe} 1$ & $178.67(18)$ & $\mathrm{H} 7 \mathrm{~A}-\mathrm{C} 7-\mathrm{H} 7 \mathrm{~B}$ & 109.5 \\
\hline $\mathrm{N} 2-\mathrm{C} 2-\mathrm{Fe} 1$ & $179.77(13)$ & $\mathrm{H} 1 \mathrm{~A}-\mathrm{O} 1-\mathrm{H} 1 \mathrm{~B}$ & $110.5(14)$ \\
\hline $\mathrm{C} 5^{\text {iv }}-\mathrm{N} 3-\mathrm{C} 5$ & $120.8(2)$ & $\mathrm{H} 1 \mathrm{~A}-\mathrm{O} 1-\mathrm{H} 1 \mathrm{~B}^{\mathrm{iv}}$ & $110.5(14)$ \\
\hline $\mathrm{C} 5^{\text {iv }}-\mathrm{N} 3-\mathrm{C} 6$ & $119.61(10)$ & $\mathrm{H} 1 \mathrm{~B}-\mathrm{O} 1-\mathrm{H} 1 \mathrm{~B}^{\mathrm{iv}}$ & $102(2)$ \\
\hline
\end{tabular}

Symmetry codes: (i) $-x,-y,-z$; (ii) $x, y,-z$; (iii) $-x,-y, z$; (iv) $x, y,-z+1$.

Hydrogen-bond geometry $\left(\AA,{ }^{\circ}\right)$

\begin{tabular}{lllll}
\hline$D-\mathrm{H} \cdots A$ & $D-\mathrm{H}$ & $\mathrm{H} \cdots A$ & $D \cdots A$ & $D-\mathrm{H} \cdots A$ \\
\hline $\mathrm{O} 1-\mathrm{H} 1 A \cdots \mathrm{N} 1^{v}$ & $0.90(3)$ & $1.67(3)$ & $2.569(2)$ & $176(3)$ \\
$\mathrm{O} 1-\mathrm{H} 1 B \cdots \mathrm{N} 2$ & $0.931(18)$ & $1.632(19)$ & $2.5589(15)$ & $173.6(18)$ \\
\hline
\end{tabular}

Symmetry code: (v) $x+1 / 2,-y+1 / 2,-z+1 / 2$. 